\title{
石杉碱甲及其类似物吡啶稠合手性双环[3.3.1]壬烷骨架的不对称构建
}

\author{
宾怀玉 程立杨小会谢建华* 周其林
}

(南开大学化学学院 元素有机化学国家重点实验室 天津 300071)

\begin{abstract}
摘要 报道了一种不对称构建石杉碱甲及其类似物吡啶稠合手性双环[3.3.1]壬烷骨架的策略. 该策略主要通过具有环 外双键的 $\gamma, \delta$-不饱和 $\beta$-酮酸酯的动态动力学拆分不对称催化氢化，并结合分子内芳基化反应为关键步骤来不对称构建 吡啶稠合手性双环 [3.3.1]壬烷骨架. 依据此策略，从已知的原料出发，经 5 步反应以 $17.4 \%$ 的总收率完成了石杉碱甲及 其类似物核心手性骨架结构的简洁、快速不对称构建.
\end{abstract}

关键词 芳基化反应; 不对称催化氢化; 双环[3.3.1]壬烷骨架；石杉碱甲；不饱和酮酸酯

\section{Enantioselective Construction of the Pyridine-Fused Chiral Bicyclo- [3.3.1]nonane Skeleton of Huperzine A and Its Analogues}

\author{
Bin, Huaiyu Cheng, Li Yang, Xiaohui Xie, Jianhua* Zhou, Qilin \\ (State Key Laboratory and Institute of Elemento-organic Chemistry, College of Chemistry, Nankai University, Tianjin 300071)
}

\begin{abstract}
A concise strategy for the enantioselective construction of the pyridine-fused chiral bicyclo[3.3.1]nonane skeleton of $(+)$-huperzine A and its nanlogues was reported. The key features of this strategy are the catalytic asymmetric hydrogenation of an exocyclic $\gamma, \delta$-unsaturated $\beta$-ketoester via dynamic kinetic resolution combined with an intramolecular arylation as the key steps to constuct the pyridine-fused chiral bicyclo[3.3.1]nonane skeleton. This strategy provides a concise and rapid approach to the chiral core structure of huperzine A and its nanlogues from the known starting materials ( 5 steps and $17.4 \%$ overall yield).
\end{abstract}

Keywords arylation; asymmetric hydrogenation; bicyclo[3.3.1]nonane skeleton; huperzine A; unsaturated ketoester

石松科生物碱(Lycopodium alkaloids)是从鰀类植物 石松科(Lycopodiaceae)和石杉科(Huperziaceae)等植物 中所分离出的结构独特的多环生物碱. 由于其具有复杂 的多环骨架结构和潜在的药用价值, 一直受到合成化学 家们的广泛关注 ${ }^{[1]}$. 石杉碱甲 [(-)-huperzine A, 图 1]是 其中最典型的代表, 它是 1986 年从我国传统中草药石 杉属植物千层塔中分离得到的具有高效、可逆、高选择 性的乙酰胆碱酷酶抑制活性的生物碱，临床已应用于治 疗阿尔茨海默症 ${ }^{[2]}$. 石杉碱甲与其类似物(一)-huperzine B、(一)-lycodine 等(图 1)均具有一个独特的含有一个手 性季碳中心的吡啶酮/吡啶稠合双环[3.3.1]壬烷手性骨 架结构, 属于 Lycodine 型石松生物碱[3]. 目前为止, 所 发现的具有乙酰胆碱脂酶抑制活性的石松生物碱大都 属于该类型生物碱, 但它们在天然植物中的含量很低.
以石杉碱甲为例, 它在石杉属植物千层塔中的含量约为

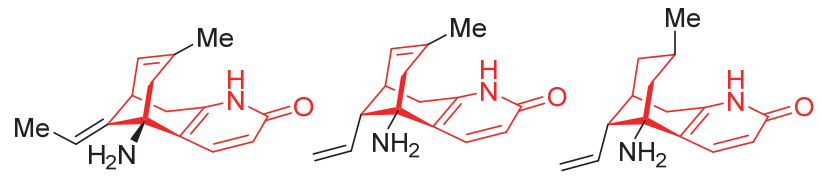

(-)-huperzine $\mathrm{A} \quad(-)$-huperzine $\mathrm{C} \quad(-)$-casuarinine $\mathrm{H}$

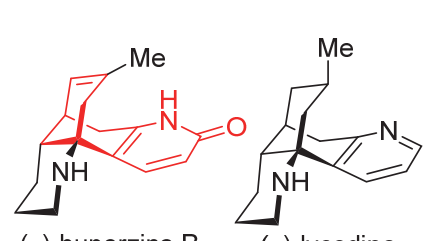

(-)-huperzine B (-)-lycodine

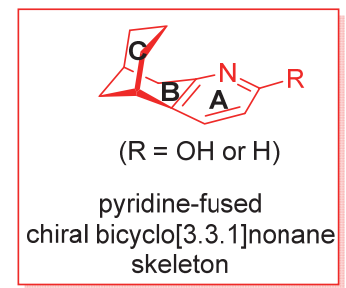

图 1 石杉碱甲及其类似物的结构

Figure 1 Structures of huperzine $A$ and its analogues

\footnotetext{
* Corresponding author. E-mail: jhxie@nankai.edu.cn

Received May 31, 2021; revised July 7, 2021; published online July 19, 2021.

Dedicated to the 100th anniversary of Chemistry at Nankai University.

Project supported by the National Natural Science Foundation of China (Nos. 21871152, 92056105, 21532003, 21790332).

国家自然科学基金(Nos. 21871152, 92056105, 21532003, 21790332)资助项目
} 
$0.01 \%$, 且千层塔植物来源不多, 生长周期长, 从而影 响了石杉碱甲在临床中的应用 ${ }^{[4]}$. 此外, 天然石杉碱甲 的活性是其对映异构体的 38 倍, 且消旋石杉碱甲难以 拆分或拆分效率低. 正因如此，石杉碱甲及其类似物的 不对称全合成研究一直受到关注, 并已发展出用不对称 催化反应为关键步骤的不对称全合成方法(Scheme 1) ${ }^{[5]}$.

(a)有机小分子催化不对称Michael-aldol串联环化反应

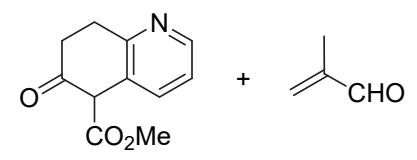

cat.*: (-)-quinine, (-)-cinchonidine, chiral guanidine, cinchona derivative
$43 \% \sim 92 \%$ yield $52 \% \sim 95 \%$ ee

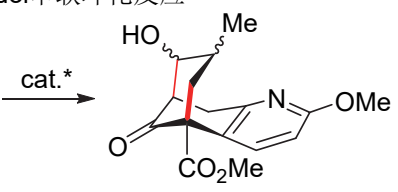

(b)钯催化不对称烯丙基烷基化串联环化反应

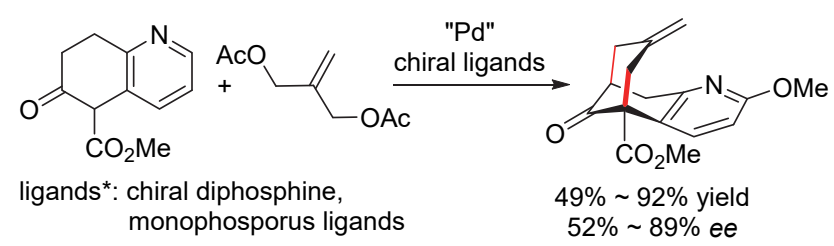

(c)钯催化不对称芳基化环化和Heck环化反应

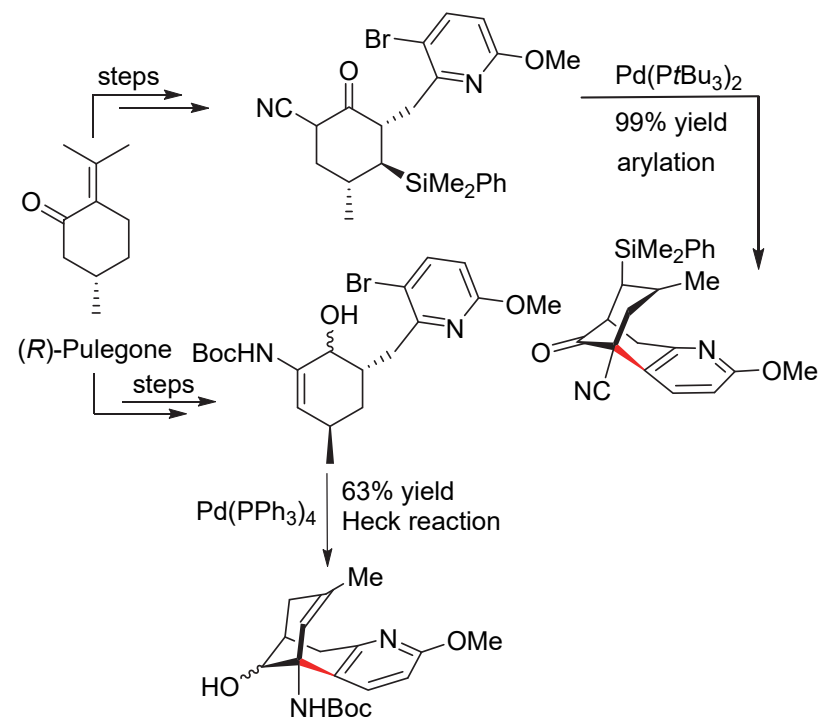

图式 1 不对称构建石杉碱甲吡啶稠合手性双环[3.3.1]壬烷骨 架的代表性策略

Scheme 1 Representative strategies for enantioselective construction of pyridine-fused chiral bicyclo[3.3.1]nonane skeleton of huperzine $\mathrm{A}$

石杉碱甲及其类似物全合成的挑战在于不对称构 建其含有手性季碳中心的吡啶酮/吡啶稠合手性双环 [3.3.1]壬烷骨架. 1989 年, 秘汝运 ${ }^{[6]}$ 和 Kozikowski 等 ${ }^{[7]}$ 分别独立报道了 2-甲氧基-6-羰基-7,8-二氢喹啉羧酸酯 与 $\alpha$-甲基丙烯醛的 Michael-aldol 串联环化为关键步骤 完成其多环手性骨架的构建, 并实现了消旋石杉碱甲的 全合成. 紧接着, 多个课题组用手性有机小分子催化剂,
如(一)-奎宁碱、(一)-辛可尼丁等, 发展了 2-甲氧基-6羰基-7,8-二氢喹啉羧酸酯与 $\alpha$-甲基丙烯醛的不对称 Michael-aldol 串联环化的不对称构建吡啶稠合手性双环 [3.3.1]壬烷骨架的策略(Scheme 1a)，并完成了(一)-石杉 碱甲的不对称全合成 ${ }^{[8]}$. 受前述工作的启发, 1993 年 Kozikowski 等 ${ }^{[9]}$ 发展了钯催化甲氧基-6-羰基-7,8-二氢喹 啉羧酸酯与 2-亚甲基丙二酸醋酸酯的双烯丙基烷基化 反应来构建消旋吡啶稠合手性双环[3.3.1]壬烷骨架. 这 一工作随后也受到了广泛的关注, 多个课题组先后用手 性磷配体的钯催化剂发展了甲氧基-6-羰基-7,8-二氢喹 啉羧酸酯与 2-亚甲基丙二酸醋酸酯的不对称烯丙基烷 基化反应来构建吡啶稠合手性双环 [3.3.1]壬烷骨架，同 样也实现了 (一)-石杉碱甲的不对称全合成 ${ }^{[10]}$ (Scheme 1b). 此外, 由于所发展的不对称催化反应为关键步骤 构建吡啶稠合手性双环 [3.3.1]壬烷骨架的效率和选择性

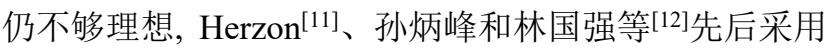
了 $(R)$-胡薄荷酮为手性起始原料, 分别发展了钯催化分 子内芳基化反应和 Heck 环化反应为关键步骤构建吡啶 稠合手性双环[3.3.1]壬烷骨架的方法(Scheme 1c)，并以 12 步 16\% 20\%的总收率和 10 步 17\% 的总收率完成了 (一)-石杉碱甲的不对称全合成. 总体而言，在过去十多 年的时间里，吡啶稠合手性双环 [3.3.1]壬烷骨架的不对 称构建得到了快速发展，合成效率和选择性得到了显著 提高, 但发展高效、高选择性的吡啶稠合手性双环 [3.3.1]壬烷骨架的不对称构建新方法和新策略仍然是 (一)-石杉碱甲及其类似物全合成研究的热点和挑战 ${ }^{[13]}$.

近年来, 我们针对复杂天然产物的手性骨架结构特 征设计发展了系列羰基化合物的高效不对称催化氢化 合成多样性手性醇的新反应，并以此为关键步骤完成了 系列天然产物分子的不对称全合成 ${ }^{[14]}$. 最近，我们在白 坚木属吲哚生物碱生源合成途径的启发下，设计发展了 具有环外双键 $\gamma, \delta$-不饱和 $\beta$-酮酸酯动态动力学拆分不对 称催化氢化合成具有两个连续手性中心手性烯丙醇的 新反应, 并以此为基础实现了白坚木属生物碱(一)goniomitine 的不对称全合成 ${ }^{[15]}$. 在此基础上，我们依据 石杉碱甲及其类似物的核心手性骨架结构特征，设计发 展了基于该高效不对称催化氢化，结合分子内芳基化反 应为关键步骤, 不对称构建吡啶稠合手性双环[3.3.1]壬 烷骨架的新策略，从而实现了石杉碱甲及其类似物多环 手性核心骨架结构的简洁、快速不对称构建.

\section{1 结果与讨论}

依据石杉碱甲及其类似物具有吡啶酮/吡啶稠合手 性双环[3.3.1]壬烷骨架结构的特征，设计了如 Scheme 2 所示的逆合成分析. 由于石杉碱甲及其类似物结构的变 
化主要集中在 $\mathrm{B} 、 \mathrm{C}$ 环上不饱和度和取代基的变化, 经 官能团转化, 设计了具有吡啶稠合手性双环[3.3.1]壬烷 骨架结构的酮酸酯 1 来合成. 酮酸酯 1 中的羰基或乙二 醇缩酮基的转化可完成 $\mathrm{B} 、 \mathrm{C}$ 环上甲基或乙基的引入和 不饱和度的修饰; 手性季碳上的氨基可用酯基为前体, 经 Curtius 重排反应引入. 依据此设想, 只需要完成酮酸 酯 1 的不对称合成就可以为石杉碱甲及其类似物的集群 式合成提供新的策略和方法. 酮酸酯 1 可由含有 3-溴-6甲氧基吡啶-2-基的环状 $\beta$-酮酸酯 2 经钯催化分子内芳 基化反应来合成. 通过分子内的手性诱导, 钯催化芳基 化反应既可构建手性季碳中心, 又可完成 C 环的构建, 其关键在于如何防止羰基 $\alpha$-位苄基取代手性中心在芳 基化反应过程中的消旋化. $\beta$-酮酸酯 2 拟通过不饱和 $\beta$ 差基酯 3 经双键的立体选择性催化氢化, 并经羟基的氧 化来合成. 不饱和 $\beta$-差基酯 3 正好可由我们已发展的具 有环外双键 $\gamma, \delta$-不饱和环状 $\beta$-酩酸酯 $\mathbf{4}$ 经动态动力学拆 分的不对称催化氢化来获得 ${ }^{[16]}$.
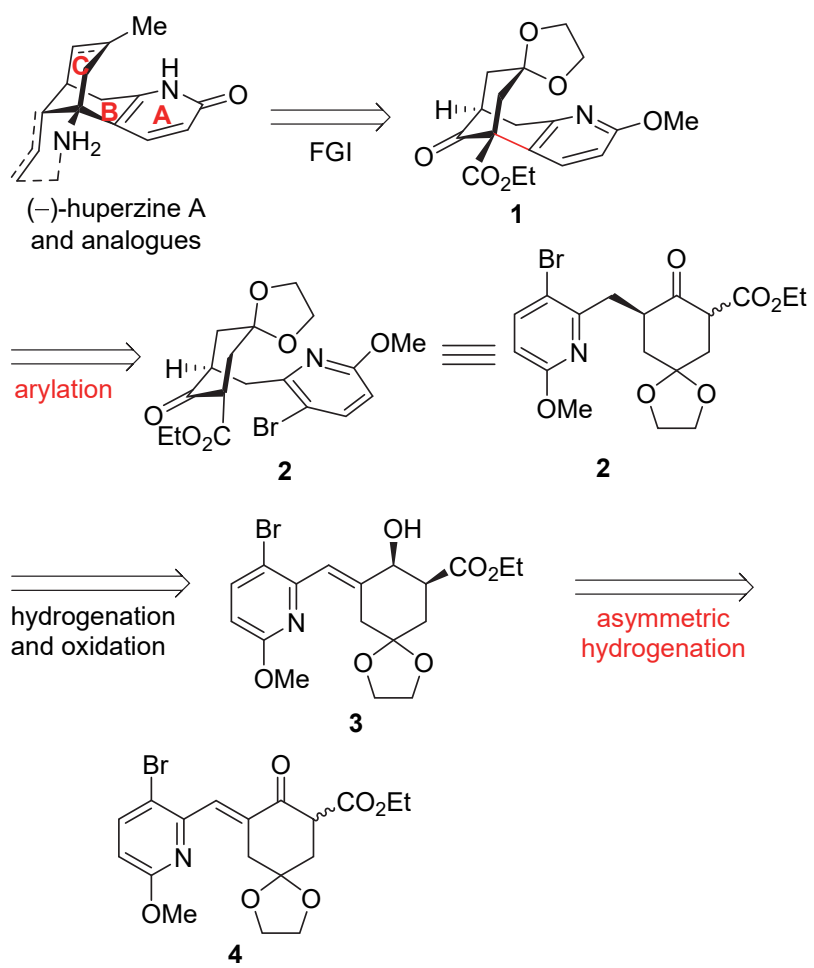

图式 2 石杉碱甲及其类似物吡啶稠合手性双环[3.3.1]壬烷骨 架不对称构建的逆合成分析

Scheme 2 Retrosynthetic analysis of enantioselective construction of the pyridine-fused chiral bicyclo[3.3.1]nonane skeleton of huperzine $\mathrm{A}$ and analogues

依据 Scheme 2 所示的逆合成分析, 先采用已发展 的具有环外双键不饱和环状 $\beta$-酮酸酯的合成方法来合 成 $\gamma, \delta$-不饱和环状 $\beta$-酮酸酯 4. 以已知的 3-溴-6-甲氧基 吡啶-2-甲醛(5)和乙二醇单缩酮保护的 2-乙氧基甲酰 基-1,4-环已二酮 6 为起始原料, 在 1,8-二氮杂二环十一
碳-7-烯(DBU)的作用下发生羟醛缩合反应，以 $49 \%$ 的分 离收率得到具有环外双键的消旋环状 $\beta$-酮酸酯 4 (Scheme 3). 接着, 选用手性螺环吡啶氨基膦配体的铱 催化剂 $\operatorname{Ir}-(R)$-SpiroPAP ${ }^{[16]}$ 在叔丁醇钾作碱的条件下，对 消旋环状 $\beta$-酮酸酯 4 进行不对称氢化，能够以 $98 \%$ 的收 率、 $98 \%$ ee 的对映选择性和 $99: 1$ 的非对映选择性得到 顺式构型的不饱和环状 $\beta$-差基酯(+)-3. 在探索不饱和 环状 $\beta$-羟基酯 $(+)-3$ 的催化氢化合成相应的不饱和 $\beta$-羟 基酯时(详细过程见辅助材料), 发现 Crabtree 催化剂

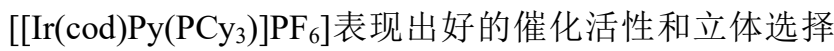
性，能以 $10: 1$ 的非对映选择性得到以顺式,反式-构型 为主的饱和环状 $\beta$-差基酯(一)-cis,trans-7, 分离收率为 91\%. 而预期希望通过立体位阻控制的产物，顺式,顺 式-构型的饱和环状 $\beta$-羟基酯(一)-cis,cis-7 的分离收率 仅为 $8 \%$ (Scheme 1). 这可能是 $\beta$-差基酯中的羟基起了 导向作用, 导致顺式, 反式-构型的氢化产物 (一)-cis, trans-7 为主. 但这不影响我们探索用(-)-cis,trans-7 经 钯催化的芳基化反应完成吡啶稠合手性双环[3.3.1]壬烷 骨架的不对称构建.

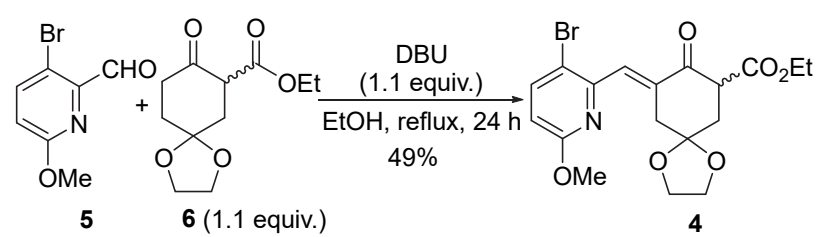

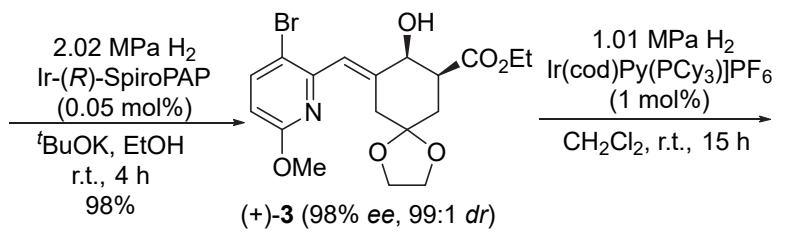

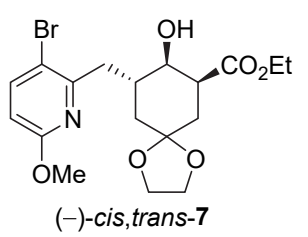
$91 \%$<smiles>CCOC1CC2(CC(Cc3nc(OC)ccc3Br)C(O)C1O)OCCO2</smiles>

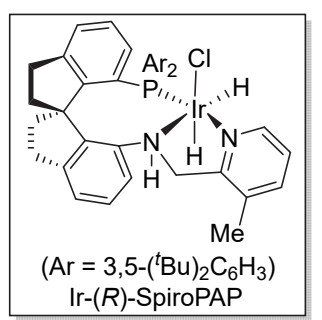

图式 3 不饱和环状 $\beta$-差基酯(+)-3 的不对称合成及其催化氢 化

Scheme 3 Enantioselective synthesis of unsaturated cyclic $\beta$-hydoxy ester ( + -) $\mathbf{3}$ and its catalytic hydrogenation 
随后, 以 $\beta$-羟基酯 $(+)$-cis,trans-7 为原料, 继续探 索氧化及钯催化分子内芳基化反应合成酮酸酯 $(+)-\mathbf{1}$, 并完成吡啶稠合手性双环 [3.3.1]壬烷骨架的不对称构建 (Scheme 4). 由于环状 $\beta$-酮酸酯(+)-2 中羰基 $\alpha$-位芐基 取代手性中心的 $\mathrm{C}-\mathrm{H}$ 具有一定的酸性, 容易在酸、碱 条件下发生消旋化. 为避免消旋化的发生, 我们经过初 步反应条件探索发现用氯铬酸吡啶盐(PCC)氧化 $\beta$-差基 酯(+)-cis,trans-7, 能够以 $95 \%$ 的分离收率得到 $\beta$-酮酸 酯(+)-2. 通过 HPLC 分析测定, 所得 $\beta$-酮酸酯 $(+)-2$ 的 光学纯度为 $96 \% e e$. 这表明在该氧化剂氧化的条件下, 羰基 $\alpha$-位苄基取代的手性中心基本保持. 值得提及的 是, 我们也尝试采用手性膦-噁唑啉配体的铱催化剂来 实现 $\beta$-酮酸酯 4 中碳碳双键的选择性氢化, 一步直接得 到 $\beta$-酮酸酯 $(+)-2^{[17]}$, 但由于底物中含有配位性的吡啶 基, 氢化反应未能发生. 在钯催化分子内芳基化反应构 建吡啶稠合手性双环[3.3.1]壬烷骨架时, 我们对反应条 件进行了系统的优化 (详细过程见辅助材料), 发现在二 (三叔丁基膦)钯 $\left(\mathrm{Pd}\left(\mathrm{P}^{t} \mathrm{Bu}_{3}\right)_{2}\right)$ 作催化剂, 磷酸钾 $\left(\mathrm{K}_{3} \mathrm{PO}_{4}\right)$ 作 碱, 以及 $110{ }^{\circ} \mathrm{C}$ 反应的条件下给出最好的结果：能以 $42 \%$ 的分离收率和 $85 \%$ ee 的光学纯度得到预期的关环 产物酮酸酯 $(+)-1$. 产物光学纯度降低的原因在于关环 反应过程使用了磷酸钾作碱, 导致 $\beta$-酩酸酯 $(+)-2$ 在关 环前发生了部分消旋化所致. 虽然在关环反应过程中, 难以避免关环产物的光学纯度降低, 但这无疑为吡啶稠 合手性双环[3.3.1]壬烷骨架的不对称构建提供了简洁、 快速的不对称构建新方法。从已知的原料出发, 只需经 5 步反应便能以 $17.4 \%$ 的总收率完成具有吡啶稠合手性 双环[3.3.1]壬烷骨架的不对称构建. 后续只需参照文献 已报道的方法, 从所合成的酮酸酯 $(+)-1$ 出发, 经官能 团转化和修饰便可完成石杉碱甲及其类似物的不对称 全合成 ${ }^{[5]}$.

\section{2 结论}

在石杉碱甲及其类似物核心手性骨架结构的启发 下, 我们设计发展了以具有环外双键 $\gamma, \delta$-不饱和环状 $\beta$ 酮酸酯动态动力学拆分不对称催化氢化, 结合分子内芳 基化反应为关键步骤的不对称构建吡啶稠合手性双环 [3.3.1]壬烷骨架的合成策略. 基于该策略, 我们从已知 的原料出发, 经羟醛缩合反应、手性螺环铱催化剂 Ir- $(R)$-SpiroPAP 催化含有 3-溴-6-甲氧基吡啶基的 $\gamma$, $\delta$-不 饱和环状 $\beta$-酮酸酯的动态动力学拆分不对称催化氢化、 铱催化双键的氢化、羟基的氧化, 以及钯催化分子内芳 基化反应，总共经 5 步反应以 17.4\%的总收率完成了吡 啶稠合手性双环 [3.3.1]壬烷手性骨架结构的不对称构 建. 该策略为石杉碱甲及其类似物多环手性骨架结构的
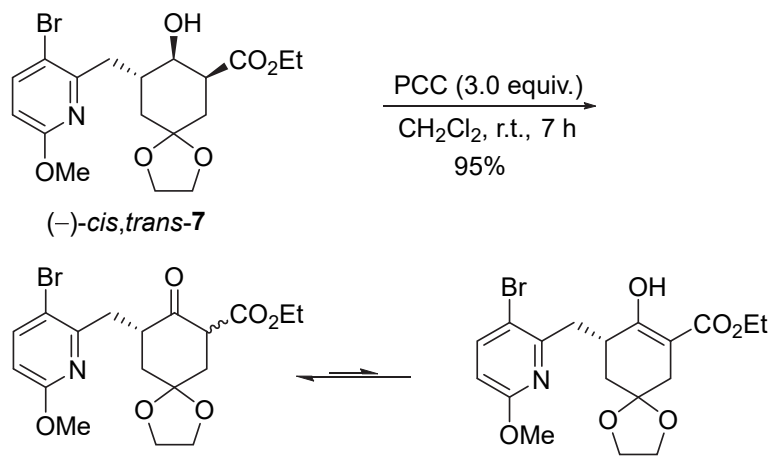

(+)-2 (96\% ee)

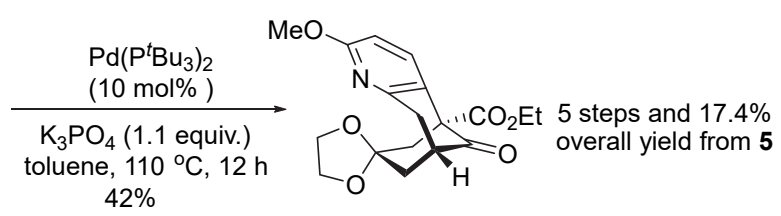

$(+)-1(85 \%$ ee $)$

图式 4 酮酸酯(+)-1 的不对称合成

Scheme 4 Enantioselective synthesis of ketoester $(+)-1$

不对称构建提供了简洁、快速的合成方法.

\section{3 实验部分}

\section{1 仪器与试剂}

${ }^{1} \mathrm{H}$ NMR 和 ${ }^{13} \mathrm{C}$ NMR 谱使用 Bruker AV-400 测定, TMS 为内标; 产物的旋光使用 Perkin Elmer 测定; 高分 辨质谱(HRMS)使用 Varian 7.0T FTMS 型仪器测定; 产 物光学纯度使用 HPLC (Hewlett Packard Model HP1100 HPLC) 测定; 熔点使用天津分析仪器厂 RY-1 型熔点仪 测定. 催化剂 $\operatorname{Ir}-(R)$-SpiroPAP 为实验室制备; 乙醇使用 镁屑回流干燥，重蒸; 四氢呋喃使用金属钠回流干燥, 重蒸; 二氯甲烷、甲苯、 $N, N$-二甲基甲酰胺和二甲亚砜 使用氢化钙回流干燥，重蒸; 其他所用试剂和溶剂均为 分析纯，直接购买使用; 柱层析使用山东省烟台市牟平 区康必诺化学试剂厂生产的 200 300 目硅胶.

\section{2 实验方法}

\section{2 .1 化合物 $\mathbf{4}$ 的合成}

往干燥的 $250 \mathrm{~mL}$ 二口烧瓶中加入 3-溴-6-甲氧基-2吡啶甲醛 $(5,6.5 \mathrm{~g}, 30.0 \mathrm{mmol})$, 并置换成氩气氛围. 加 入 4,4-(乙二氧基)-2-乙氧羰基环已酮 $(6,5.6 \mathrm{~g}, 33.0$ $\mathrm{mmol}, 5.3 \mathrm{~mL})$ 以及乙醇 $(100 \mathrm{~mL})$, 并搅拌均匀. 加入 DBU $(5.0 \mathrm{~g}, 33.0 \mathrm{mmol}, 5.0 \mathrm{~mL})$, 回流摚拌反应. 使用薄 层色谱(TLC)监测反应进行情况. $24 \mathrm{~h}$ 后反应结束, 反应 体系降温到 $0{ }^{\circ} \mathrm{C}$, 加入饱和氯化铵溶液 $(100 \mathrm{~mL})$ 淬灭反 应，乙酸乙酯萃取 $(100 \mathrm{~mL} \times 3)$. 合并有机相并用饱和氯 化钠溶液 $(100 \mathrm{~mL})$ 洗涤，无水硫酸钠干燥，抽滤除去干 燥剂, 浓缩得到粗产品. 使用适量二氯甲烷溶解粗产品, 并加入适量硅胶混合均匀, 减压脱溶, 干法上柱, 硅胶 
柱层析(石油醚/乙酸乙酯体积比由 $20 ： 1$ 到 $10 ： 1$ ), 得 到(E)-9-(3-溴-6-甲氧基-2-吡啶)亚甲基-8-氧代-1,4-二噁 螺[4.5]癸烷-7-羧酸乙酯(4) (6.3 g, $14.7 \mathrm{mmol})$, 为淡黄色 固体, 收率为 $79 \%$. m.p. $140 \sim 141{ }^{\circ} \mathrm{C}, R_{\mathrm{f}}=0.57$ (石油醚) 乙酸乙酯, $V: V=5: 2) .{ }^{1} \mathrm{H}$ NMR $\left(400 \mathrm{MHz}, \mathrm{CDCl}_{3}\right) \delta$ : $12.42(\mathrm{~s}, 1 \mathrm{H}), 7.79(\mathrm{~s}, 1 \mathrm{H}), 7.71(\mathrm{~d}, J=8.7 \mathrm{~Hz}, 1 \mathrm{H}), 6.54$ (d, $J=8.7 \mathrm{~Hz}, 1 \mathrm{H}), 4.27(\mathrm{q}, J=7.1 \mathrm{~Hz}, 2 \mathrm{H}), 4.06 \sim 3.92$ (m, 4H), 3.92 (s, 3H), 3.41 (d, $J=2.2 \mathrm{~Hz}, 2 \mathrm{H}), 2.68$ (s, $2 \mathrm{H}), 1.33$ (t, $J=7.1 \mathrm{~Hz}, 3 \mathrm{H}) ;{ }^{13} \mathrm{C}$ NMR $\left(101 \mathrm{MHz}, \mathrm{CDCl}_{3}\right)$ $\delta: 172.4,164.0,161.7,150.4,142.9,134.6,127.4,114.8$, 111.6, 107.4, 99.2, 64.7, 67.0, 53.9, 36.7, 33.2, 14.3. HRMS (ESI) calcd for $\mathrm{C}_{18} \mathrm{H}_{21} \mathrm{BrNO}_{6}[\mathrm{M}+\mathrm{H}]^{+} 426.0552$; found 426.0542 .

\subsection{2 化合物 $(+)-3$ 的合成}

在手套箱中分别称取催化剂 Ir- $(R)$-SpiroPAP (5.0 $\mathrm{mg}, 0.005 \mathrm{mmol}$ )和叔丁醇钾 $(5.6 \mathrm{mg}, 0.05 \mathrm{mmol})$ 于两个 干燥的 $15 \mathrm{~mL}$ Schlenk 管中, 取出手套箱后分别向两个 Schlenk 管中加入无水乙醇 $(5 \mathrm{~mL})$ 将固体化合物溶解. 在 $250 \mathrm{~mL}$ 氢化反应内管中称取化合物 $4(4.3 \mathrm{~g}, 20.0$ $\mathrm{mmol}$ )后, 将反应内管放入氢化反应釜中, 并将体系置 换为氩气氛围, 用注射器快速向氢化釜的反应内管中依 次加入无水乙醇 $(30 \mathrm{~mL})$ 、催化剂溶液及碱溶液, 快速将 体系置换为氢气氛围并充氢气至 $2.02 \mathrm{MPa}$, 室温 $\left(25{ }^{\circ} \mathrm{C}\right)$ 下搅拌反应至没有氢气压力降, 大约反应 $4 \mathrm{~h}$. 缓慢释放氢气, 加入饱和氯化铵溶液 $(40 \mathrm{~mL})$ 淬灭反应, 乙酸乙酯萃取 $(50 \mathrm{~mL} \times 3)$. 合并有机相并用饱和氯化钠 溶液 $(50 \mathrm{~mL})$ 洗涤, 无水硫酸钠干燥, 抽滤除去干燥剂, 浓缩得到粗产品. 用适量二氯甲烷溶解粗产品, 并加入 适量硅胶混合均匀, 减压脱溶, 干法上柱, 硅胶柱层析 (石油醚/乙酸乙酯体积比由 $10: 1$ 到 $5: 1$ ), 得到(+)cis-(E)-9-(3-澳-6-甲氧基-2-吡啶)亚甲基-8-羟基-1,4-二噁 螺[4.5]癸烷-7-羧酸乙酯 $[(+)-3](4.2 \mathrm{~g}, 9.8 \mathrm{mmol})$, 白色 固体粉末, 收率为 $98 \%, 98 \%$ ee. m.p. $43 \sim 44{ }^{\circ} \mathrm{C}, R_{\mathrm{f}}=$ 0.32 (石油醚/乙酸乙酯, $V: V=5: 2$ ), $[\alpha]_{\mathrm{D}}^{25}+122.9$ (c 1.0 $\mathrm{CHCl}_{3}$ ). 高效液相色谱分离条件: Chiralcel AS-3 column $(25 \mathrm{~cm} \times 0.46 \mathrm{~cm} \mathrm{ID})$; 正己烷/异丙醇 $(V: V=85: 15)$; 温度: $25{ }^{\circ} \mathrm{C}$; 流速: $1.0 \mathrm{~mL} / \mathrm{min}$ : 检测波长: $254 \mathrm{~nm}$; $t_{\mathrm{R}}($ minor $)=21.10 \mathrm{~min}, t_{\mathrm{R}}($ major $)=13.70 \mathrm{~min} .{ }^{1} \mathrm{H}$ NMR $\left(400 \mathrm{MHz}, \mathrm{CDCl}_{3}\right) \delta: 7.66(\mathrm{~d}, J=8.7 \mathrm{~Hz}, 1 \mathrm{H}), 6.88(\mathrm{~d}, J=$ $2.4 \mathrm{~Hz}, 1 \mathrm{H}), 6.50(\mathrm{~d}, J=8.7 \mathrm{~Hz}, 1 \mathrm{H}), 4.66(\mathrm{~s}, 1 \mathrm{H}), 4.22(\mathrm{q}$, $J=7.2 \mathrm{~Hz}, 2 \mathrm{H}), 4.07 \sim 3.84(\mathrm{~m}, 8 \mathrm{H}), 3.05 \sim 2.88(\mathrm{~m}, 2 \mathrm{H})$, $2.83(\mathrm{~d}, J=14.1 \mathrm{~Hz}, 1 \mathrm{H}), 2.37$ (t, $J=12.9 \mathrm{~Hz}, 1 \mathrm{H}), 1.97$ $(\mathrm{d}, J=12.9 \mathrm{~Hz}, 1 \mathrm{H}), 1.30(\mathrm{t}, J=7.2 \mathrm{~Hz}, 3 \mathrm{H}) ;{ }^{13} \mathrm{C}$ NMR $\left(101 \mathrm{MHz}, \mathrm{CDCl}_{3}\right) \delta: 173.7,161.7,150.3,143.6,142.8$, 124.8, 113.0, 111.0, 108.8, 73.5, 64.6, 64.5, 60.9, 53.8,
45.2, 33.9, 31.5, 14.2. HRMS (ESI) calcd for $\mathrm{C}_{18} \mathrm{H}_{23} \mathrm{BrNO}_{6}$ $[\mathrm{M}+\mathrm{H}]^{+}$428.0709; found 428.0698.

\subsection{3 化合物 (一)-cis,trans-7 的合成}

往 $25 \mathrm{~mL}$ 氢化内管中加入 $\left[\operatorname{Ir}(\operatorname{cod}) \mathrm{Py}\left(\mathrm{PCy}_{3}\right)\right] \mathrm{PF}_{6}$ (16.1 mg, $0.02 \mathrm{mmol})$, 将反应内管放入氢化反应釜中, 并将体系置换为氩气氛围. 随后用注射器快速向氢化釜 的氢化内管中加入 $(+)-3(857 \mathrm{mg}, 2.0 \mathrm{mmol})$ 的二氯甲烷 $(12 \mathrm{~mL})$ 溶液, 并将体系置换为氢气氛围并充氢气至 $1.01 \mathrm{MPa}$, 室温 $\left(25{ }^{\circ} \mathrm{C}\right)$ 下搅拌反应 $24 \mathrm{~h}$. 反应结束后, 缓慢释放氢气, 硅藻土抽滤, 滤液加入适量硅胶混合均 匀, 减压脱溶, 干法上柱, 硅胶柱层析 (正己烷/乙醚, $V: V=3: 2$ )得到化合物(一)-cis,trans-7 (783 mg, 1.82 $\mathrm{mmol}$ )和化合物(一)-cis, cis-7 (69 mg, $0.16 \mathrm{mmol})$.

(-)-cis,trans-9-(3-溴-6-甲氧基-2-吡啶)甲基-8-羟 基-1,4-二噁螺[4.5]癸烷-7-羧酸乙酯[(-)-cis,trans-7]: 无 色粘稠油状液体, 收率为 $91 \% . R_{\mathrm{f}}=0.39$ (石油醚/乙酸乙 酯, $V: V=1: 1),[\alpha]_{\mathrm{D}}^{25}-16.0\left(c 1.0 \mathrm{CHCl}_{3}\right) ;{ }^{1} \mathrm{H}$ NMR $\left(400 \mathrm{MHz}, \mathrm{CDCl}_{3}\right) \delta: 7.61(\mathrm{~d}, J=8.6 \mathrm{~Hz}, 1 \mathrm{H}), 6.47(\mathrm{~d}, J=$ $8.6 \mathrm{~Hz}, 1 \mathrm{H}), 4.19(\mathrm{q}, J=7.1 \mathrm{~Hz}, 2 \mathrm{H}), 4.02 \sim 3.96(\mathrm{~m}, 1 \mathrm{H})$, $3.94 \sim 3.82(\mathrm{~m}, 6 \mathrm{H}), 3.80 \sim 3.72(\mathrm{~m}, 1 \mathrm{H}), 3.51(\mathrm{~s}, 1 \mathrm{H})$, $3.26(\mathrm{dd}, J=13.8,6.1 \mathrm{~Hz}, 1 \mathrm{H}), 3.07(\mathrm{dt}, J=8.0,3.9 \mathrm{~Hz}$, $2 \mathrm{H}), 2.92 \sim 2.75(\mathrm{~m}, 2 \mathrm{H}), 2.27$ (dd, $J=13.1,8.3 \mathrm{~Hz}, 2 \mathrm{H})$, 1.97 (dd, $J=13.7,4.7 \mathrm{~Hz}, 1 \mathrm{H}), 1.80$ (dd, $J=13.4,4.8 \mathrm{~Hz}$, 1H), 1.56 (dd, $J=13.6,6.7 \mathrm{~Hz}, 1 \mathrm{H}), 1.28(\mathrm{t}, J=7.1 \mathrm{~Hz}$, $3 \mathrm{H}) ;{ }^{13} \mathrm{C}$ NMR $\left(101 \mathrm{MHz}, \mathrm{CDCl}_{3}\right) \delta: 174.8,162.2,156.0$, 142.4, 112.2, 109.9, 108.0, 71.7, 64.5, 64.1, 60.6, 53.6, $42.8,38.9,38.2,36.4,33.3,14.1$. HRMS (ESI) calcd for $\mathrm{C}_{18} \mathrm{H}_{25} \mathrm{BrNO}_{6}[\mathrm{M}+\mathrm{H}]^{+}$430.0865; found 430.0862 .

(一)-cis,cis-9-(3-澳-6-甲氧基-2-吡啶)甲基-8-羟基1,4-二噁螺 [4.5]癸烷-7-羧酸乙酯[(一)-cis,cis-7]: 无色粘 稠油状液体, 收率为 $8 \% . R_{\mathrm{f}}=0.44$ (石油醚/乙酸乙酯, $V: V=1: 1),[\alpha]_{\mathrm{D}}^{25}-17.4\left(c 1.0, \mathrm{CHCl}_{3}\right) ;{ }^{1} \mathrm{H}$ NMR $(400$ $\left.\mathrm{MHz}, \mathrm{CDCl}_{3}\right) \delta: 7.66(\mathrm{~d}, J=8.7 \mathrm{~Hz}, 1 \mathrm{H}), 6.52(\mathrm{~d}, J=8.7$ $\mathrm{Hz}, 1 \mathrm{H}), 4.21 \sim 4.10(\mathrm{~m}, 2 \mathrm{H}), 4.04 \sim 3.90(\mathrm{~m}, 5 \mathrm{H}), 3.87(\mathrm{~s}$, $3 \mathrm{H}), 3.45$ (s, 1H), 3.01 (dd, $J=14.0,6.1 \mathrm{~Hz}, 1 \mathrm{H}), 2.90$ (dd, $J=13.9,9.2 \mathrm{~Hz}, 1 \mathrm{H}), 2.75-2.67(\mathrm{~m}, 1 \mathrm{H}), 2.37 \sim 2.28(\mathrm{~m}$, $1 \mathrm{H}), 2.10(\mathrm{t}, J=13.3 \mathrm{~Hz}, 1 \mathrm{H}), 1.91 \sim 1.81(\mathrm{~m}, 2 \mathrm{H}), 1.61 \sim$ $1.55(\mathrm{~m}, 1 \mathrm{H}), 1.24(\mathrm{t}, J=7.1 \mathrm{~Hz}, 3 \mathrm{H}) ;{ }^{13} \mathrm{C}$ NMR $(101$ $\left.\mathrm{MHz}, \mathrm{CDCl}_{3}\right) \delta: 173.9,162.3,155.1,142.8,112.1,110.5$, 108.5, 67.0, 64.4, 64.4, 60.6, 53.6, 45.3, 39.1, 38.2, 35.3, 30.9, 14.2. HRMS (ESI) calcd for $\mathrm{C}_{18} \mathrm{H}_{25} \mathrm{BrNO}_{6}[\mathrm{M}+\mathrm{H}]^{+}$ 430.0865; found 430.0863 .

\section{2 .4 化合物 $(+)-2$ 的合成}

往 $50 \mathrm{~mL}$ 圆底烧瓶中加入化合物(-)-cis,trans-7 (427 $\mathrm{mg}, 1.0 \mathrm{mmol})$ 的二氯甲烷 $(10 \mathrm{~mL})$ 溶液, 搅拌均匀, 分批 
加入氯铬酸吡啶盐 $(647 \mathrm{mg}, 3.0 \mathrm{mmol})$. 室温下搅拌 $7 \mathrm{~h}$. 反应结束, 硅藻土抽滤, 滤液加入适量硅胶混合均匀, 减压脱溶, 干法上柱, 硅胶柱层析(石油醚/乙酸乙酯, $V: V=5: 1$ )得到(+)-9-(3-溴-6-甲氧基-2-吡啶)甲基-8氧代-1,4-二啞螺[4.5]癸烷-7-羧酸乙酯[(+)-2] (407 mg, $0.95 \mathrm{mmol})$, 为无色粘稠油状液体, 收率为 $95 \%, 96 \%$ ee. $R_{\mathrm{f}}=0.45$ (石油醚 $/ 乙$ 酸乙酯, $V: V=5: 2$ ), m.p. 43 $44{ }^{\circ} \mathrm{C} ;[\alpha]_{\mathrm{D}}^{25}+37.0$ (c $\left.1.0 \mathrm{CHCl}_{3}\right)$. 高效液相色谱分离条 件: Chiralcel OD-H column $(25 \mathrm{~cm} \times 0.46 \mathrm{~cm} \mathrm{ID})$; 正己 烷/异丙醇 $(V: V=95: 5)$; 温度: $25{ }^{\circ} \mathrm{C}$; 流速: $1.0 \mathrm{~mL} /$ $\min$; 检 测 波长: $254 \mathrm{~nm} ; t_{\mathrm{R}}$ (minor) $=5.61 \mathrm{~min}$; $t_{\mathrm{R}}($ major $)=5.93 \mathrm{~min} .{ }^{1} \mathrm{H} \mathrm{NMR}\left(400 \mathrm{MHz}, \mathrm{CDCl}_{3}\right) \delta: 12.63$ (s), $7.70 \sim 7.58(\mathrm{~m}), 6.53 \sim 6.40(\mathrm{~m}), 4.29 \sim 4.13(\mathrm{~m})$, $4.08 \sim 3.90(\mathrm{~m}), 3.89 \sim 3.82(\mathrm{~m}), 3.68 \sim 3.57(\mathrm{~m}), 3.51 \sim$ $3.38(\mathrm{~m}), 3.33(\mathrm{dd}, J=16.4,6.6 \mathrm{~Hz}), 3.13(\mathrm{dd}, J=14.6,9.0$ $\mathrm{Hz}), 2.88(\mathrm{dd}, J=15.4,8.8 \mathrm{~Hz}), 2.74(\mathrm{dd}, J=16.5,6.7 \mathrm{~Hz})$, $2.63(\mathrm{dt}, J=13.9,3.7 \mathrm{~Hz}), 2.58 \sim 2.47(\mathrm{~m}), 2.44 \sim 2.33$ (m), $2.28 \sim 2.18(\mathrm{~m}), 2.15 \sim 2.03(\mathrm{~m}), 2.02 \sim 1.88(\mathrm{~m}), 1.78$ $(\mathrm{d}, J=8.0 \mathrm{~Hz}, 2 \mathrm{H}), 1.36 \sim 1.23(\mathrm{~m}) ;{ }^{13} \mathrm{C} \mathrm{NMR}(101 \mathrm{MHz}$, $\left.\mathrm{CDCl}_{3}\right) \delta: 205.5,173.3,172.4,169.2,162.3,162.1,154.7$, $154.0,142.3,142.2,112.3,112.1,110.0,109.9,107.1$, $106.8,95.1,64.9,64.7,64.5,64.4,61.1,60.5,53.9,53.5$, 53.5, 45.1, 41.0, 37.7, 37.4, 37.1, 35.5, 35.2, 33.2, 14.3, 14.1. HRMS (ESI) calcd $\mathrm{C}_{18} \mathrm{H}_{23} \mathrm{BrNO}_{6}[\mathrm{M}+\mathrm{H}]{ }^{+}$ 428.0709; found 428.0703.

\section{2 .5 化合物 $(+)-1$ 的合成}

往 $15 \mathrm{~mL}$ 的 Schlenk 管中称取入化合物 $(+)-2$ (50.0 $\mathrm{mg}, 0.12 \mathrm{mmol}) 、 \mathrm{Pd}\left(\mathrm{P}^{t} \mathrm{Bu}_{3}\right)_{2}(6.0 \mathrm{mg}, 0.01 \mathrm{mmol})$ 和 $\mathrm{K}_{3} \mathrm{PO}_{4}$ $(27.3 \mathrm{mg}, 0.13 \mathrm{mmol})$, 置换为氮气氛围, 在另一个 15 $\mathrm{mL}$ 的 Schlenk 管中加入 $3 \mathrm{~mL}$ 甲苯, 反复脱气三次后转 移到体系中, $110{ }^{\circ} \mathrm{C}$ 下加热搅拌反应 $12 \mathrm{~h}$. 反应结束后, 将反应体系冷却至室温, 硅藻土抽滤, 滤液加入适量硅 胶混合均匀, 减压脱溶, 干法上柱, 硅胶柱层析(甲苯/ 乙酸乙酯, $V: V=7: 1)$, 得到 $(+)$-2'-甲氧基-11'-氧代9',10'-二氢-6' $H$-螺 [ $[1,3]$-二氧烷-2,7'-[5,9]甲撑环辛并 $[b]$ 吡啶]-5'(8'H)-羧酸乙酯[(+)-1] (17.0 mg, $0.05 \mathrm{mmol})$, 为 无色油状液体, 收率为 $42 \%, 85 \% e e . \quad R_{\mathrm{f}}=0.36$ (石油 醚/乙酸乙酯, $V: V=1: 1),[\alpha]_{\mathrm{D}}^{25}+1.3\left(c 1.0 \mathrm{CHCl}_{3}\right)$. 高 效液相色谱分离条件：Chiralcel OD-H column (25 $\mathrm{cm} \times 0.46 \mathrm{~cm} \mathrm{ID)}$; 正已烷/异丙醇 $(V: V=90: 10)$; 温 度： $25{ }^{\circ} \mathrm{C}$; 流速： $1.0 \mathrm{~mL} / \mathrm{min}$; 检测波长： $254 \mathrm{~nm}$; $t_{\mathrm{R}}($ major $)=9.08 \mathrm{~min}, t_{\mathrm{R}}($ minor $)=10.05 \mathrm{~min} .{ }^{1} \mathrm{H}$ NMR $(400$ $\left.\mathrm{MHz}, \mathrm{CDCl}_{3}\right) \delta: 7.00(\mathrm{~d}, J=8.8 \mathrm{~Hz}, 1 \mathrm{H}), 6.57(\mathrm{~d}, J=8.8$ $\mathrm{Hz}, 1 \mathrm{H}), 4.27$ (q, $J=7.2 \mathrm{~Hz}, 2 \mathrm{H}), 3.92$ (s, 3H), 3.81 3.71 $(\mathrm{m}, 3 \mathrm{H}), 3.65 \sim 3.58(\mathrm{~m}, 1 \mathrm{H}), 3.48(\mathrm{dd}, J=18.0,7.6 \mathrm{~Hz}$,
1H), $3.26(\mathrm{~d}, J=18.0 \mathrm{~Hz}, 1 \mathrm{H}), 3.00 \sim 2.94(\mathrm{~m}, 1 \mathrm{H}), 2.79$ $(\mathrm{d}, J=14.0 \mathrm{~Hz}, 1 \mathrm{H}), 2.41(\mathrm{dd}, J=14.0,5.6 \mathrm{~Hz}, 1 \mathrm{H}), 2.28$ $(\mathrm{dd}, J=14.2,3.6 \mathrm{~Hz}, 1 \mathrm{H}), 2.22 \sim 2.14(\mathrm{~m}, 1 \mathrm{H}), 1.24(\mathrm{t}, J=$ $7.2 \mathrm{~Hz}, 3 \mathrm{H}) ;{ }^{13} \mathrm{C}$ NMR (101 MHz, $\left.\mathrm{CDCl}_{3}\right) \delta: 208.3,170.2$, $162.8,151.6,137.8,124.9,108.8,106.3,64.8,63.4,61.7$, 59.6, 53.4, 46.3, 43.7, 43.6, 40.1, 14.1. HRMS (ESI) calcd for $\mathrm{C}_{15} \mathrm{H}_{18} \mathrm{O}_{3} \mathrm{Na}[\mathrm{M}+\mathrm{Na}]^{+}$370.1262, found 370.1258 .

辅助材料(Supporting Information) 反应条件优化实 验、合成化合物的 ${ }^{1} \mathrm{H} N \mathrm{NMR}$ 和 ${ }^{13} \mathrm{C} \mathrm{NMR}$ 的谱图. 这些材 料可以免费从本刊网站(http://sioc-journal.cn/)上下载.

\section{References}

[1] (a) Siengalewicz, P.; Mulzer, J.; Rinner, U. In The Alkaloids: Chemistry and Biology, Ed.: Knölker, H.-J., Academic Press, Cambridge, MA, 2013, Vol. 72, pp. 1 151.

(b) Ma, X. Q.; Gang, D. R. Nat. Prod. Rep. 2004, 21, 752.

(c) Xiao, C.; Gao, L.; Wang, J.; Miao, Y.; Fan, H. Chin. J. Org. Chem. 2017, 37, 810 (in Chinese).

(肖春霞, 曹林, 王佳, 苗银龙, 范华芳, 有机化学, 2017, 37, 810.)

[2] (a) Herzon, S. B.; Tun, M. K. M. J. Exp. Pharmacol. 2012, 4, 113. (b) Qian, Z. M.; Ke, Y. Front. Aging Neurosci. 2014, 6, 216.

[3] (a) Wang, L.-L.; Hao, L.-J.; Zhou, Z.-B.; Zhu, X.-L.; Shi, Z.-H.; Miyamoto, T.; Pan, K. Phytochemistry 2018, 154, 63.

(b) Zhang, D.-B.; Chen, J.-J.; Song, Q.-Y.; Zhang, L.; Gao, K. Molecules 2014, 19, 9999.

(c) Tang, Y.; Fu, Y.; Xiong, J.; Li, M.; Ma, G.-L.; Yang, G.-X.; Wei, B.-G.; Zhao, Y.; Zhang, H.-Y.; Hu, J.-F. J. Nat. Prod. 2013, 76, 1475.

(d) Yin, S.; Fan, C.-Q.; Wang, X.-N.; Yue, J.-M. Helv. Chem. Acta 2006, 89, 138.

[4] (a) Bai, D.-L.; Tang, X.-C.; He, X.-C. Curr. Med. Chem. 2000, 7 , 355.

(b) Ma, X.; Tan, C.; Zhu, D.; Gang, D.-R. J. Ethnopharmacol. 2006, 104, 54

[5] Zheng, S.; Yu, C.; Shen, Z. Chin. J. Org. Chem. 2013, 33, 2261 (in Chinese).

(郑书岩, 郁春辉, 沈征武, 有机化学, 2013, 33, 2261.)

[6] Qian, L.-G.; Ji, R.-Y. Tetrahedron Lett. 1989, 30, 2089.

[7] Xia, Y.; Kozikowshi, A. P. J. Am. Chem. Soc. 1989, 111, 4166.

[8] (a) Chen, W.-P.; Yang, F.-Q. Chin. J. Med. Chem. 1995, 15, 10 (in Chinese).

(陈卫平，杨福秋，中国药物化学杂志, 1995, 15, 10.)

(b) Kaneko, S.; Yoshino, T.; Katoh, T.; Terashima, S. Heterocycles 1997, 46, 27

(c) Kaneko, S.; Yoshino, T.; Katoh, T.; Terashima, S. Tetrahedron 1998, 54, 5471.

(d) Pan, Q.-B.; Ma, D.-W. Chin. J. Chem. 2003, 21, 793.

(e) Ding, X.-H.; Li, X.; Liu, D.; Cui, W.-C.; Ju, X.; Wang, S.-Z.; Yao, Z.-J. Tetrahedron 2012, 68, 6240.

[9] (a) Kozikowski, A.P.; Campiani, G.; Aagaard, P.; Mckinney, M. J. Chem. Soc., Chem. Commun. 1993, 10, 860.

(b) Campiano, G.; Sun, L. Q.; Kozikowski, A. P. J. Org. Chem 1993, 58, 7660 .

[10] (a) Kaneko, S.; Yoshino, T.; Katoh, T.; Terashima, S. Tetrahedron: Asymmetry 1997, 8, 829.

(b) He, X.-C.; Wang, B.; Bai, D.-L. Tetrahedron Lett. 1998, 39, 411. (c) Chassaing, C.; Hanudrechy, A.; Langlois, Y. Tetrahedron Lett. 1999, 40, 8805 .

(d) He, X.-C.; Wang, B.; Yu, G, Bai, D. Tetrahedron: Asymmetry 2001, 12, 3213 . 
(e) Tudhope, S. R.; Bellamy, J. A.; Ball, A.; Rajasekar, D.; Azadi-Ardakani, M.; Meera, H. S.; Gnanadeepam, J. M.; Saiganesh, R.; Gibson, F.; He, L.; Behrens, C. H.; Underiner, G.; Marfurt, J.; Favre, N. Org. Process Res. Dev. 2012, 16, 635.

(f) Lin, C.-F.; Chien, C.-W.; Ojima, I. Org. Chem. Front. 2014, 1, 1062 .

[11] Tun, M. K. M.; Wüstmann, D.; Herzon, S. B. Chem. Sci. 2011, 2, 2251.

[12] (a) Ding, R.; Sun, B.-F.; Lin, G.-Q. Org. Lett. 2012, 14, 4446.

(b) Ding, R.; Fu, J.-G.; Xu, G.-Q.; Sun, B.-F.; Lin, G.-Q. J. Org. Chem. 2014, 79, 240.

(c) Fu, J.-G.; Xu, G.-Q.; Ding, R.; Lin, G.-Q.; Sun, B.-F. Org. Chem. Front. 2016, 3, 62.

[13] Haley, H. M. S.; Payer, S. E.; Papiddocha, S. M.; Clemens, S.; Nyenhuis, J.; Sarpong, R. J. Am. Chem. Soc. 2021, 143, 4732.

[14] (a) Xie, J.-H.; Zhou, Q.-L. Aldrichim. Acta 2015, 48, 33.

(b) Lin, H.; Xiao, L.-J.; Zhou, M.-J.; Yu, H.-M.; Xie, J.-H.; Zhou, Q.-L. Org. Lett. 2016, 18, 1434.

(c) Liu, Y.; Cheng, L.-J.; Yue, H.-T.; Che, W.; Xie, J.-H.; Zhou, Q.-L. Chem. Sci. 2016, 6, 4725.

(d) Zou, X.-D.; Gou, S.-M.; Yang, R.; Xie, J.-H.; Zhou, Q.-L. Chem. Sci. 2017, 8, 6202.

(e) Liu, Y.-T.; Li, L.-P.; Xie, J.-H.; Zhou, Q.-L. Angew. Chem., Int. Ed. 2017, 56, 12708 . (f) Zou, X.-D.; Gou, S.-M.; Yang, R.; Xie, J.-H.; Zhou, Q.-L. Org. Lett. 2017, 19, 5240 .

(g) Yang, X.-H.; Gu, X.-S.; Bin, H.-Y; Xie, J.-H.; Zhou, Q.-L. Chin. J. Org. Chem. 2020, 40, 3963 (in Chinese).

(杨小会，顾雪松，宾怀玉，谢建华，周其林，有机化学，2020, 40, 3963.)

[15] (a) Bin, H.-Y.; Wang, K.; Yang, D.; Yang, X.-H.; Xie, J.-H.; Zhou, Q.-L. Angew. Chem., Int. Ed. 2019, 58, 1174.

(b) Bin, H.-Y.; Cheng, L.; Wu, X.; Zhu, C.-L.; Yang, X.-H.; Xie, J.-H.; Zhou, Q.-L. Chem. Sci. 2021, 12, 7793.

[16] (a) Xie, J.-H.; Zhou, Q.-L. Acta Chim. Sinica 2014, 72, 778 (in Chinese).

(谢建华, 周其林, 化学学报, 2014, 72, 778.)

(b) Yang, X.-H.; Xie, J.-H.; Zhou, Q.-L. Org. Chem. Front. 2014, 1, 190.

(c) Xie, J.-H.; Liu, X.-Y.; Xie, J.-B.; Wang, L.-X.; Zhou, Q.-L. Angew. Chem., Int. Ed. 2011, 50, 7329.

[17] (a) Lu, S.-M.; Bolm, C. Angew. Chem., Int. Ed. 2008, 47, 8920.

(b) Lu, W.-J.; Chen, Y.-W.; Hou, X.-L. Angew. Chem., Int. Ed. 2008, 47, 10133

(c) Tian, F.; Yao, D.; Liu, Y.; Xie, F.; Zhang, W. Adv. Synth. Catal. 2010, 352, 1841 .

(d) Liu, X.; Han, Z.; Wang, Z.; Ding, K. Angew. Chem., Int. Ed. 2014, 53, 1978. 